\title{
Adrenomyeloneuropathie, eine seltene Ursache der primären Nebennierenrindeninsuffizienz
}

\author{
K. Frank, O. Schrecker, K. Brosi, K. H. Krause, P. Vescei, H. W. Moser und R. Ziegler \\ Abteilung Innere Medizin VI-Endokrinologie -, Neurologische Klinik und Pharmakologisches Institut der Universität Heidelberg, sowie J. F. Kennedy Institute for Handicapped \\ Children, Baltimore/Maryland
}

\begin{abstract}
Eine seltene Ursache der primären Nebennierenrindeninsuffizienz ist die X-chromosomal vererbte Adrenomyeloneuropathie. Neurologisch ist sie durch Zeichen der zentralen und peripheren Demyelinisierung, endokrinologisch durch einen M. Addison und eine zusätzliche primäre Hodeninsuffizienz gekennzeichnet. Bei zwei Patienten mit diesem Krankheitsbild konnte der Stoffwechseldefekt im Abbau langkettiger Fettsäuren durch Bestimmung eines erhöhten HexakosansäureSpiegels (C 26) im Blut gesichert werden. Bei einem Patienten (Fall 1) lag ein isolierter Ausfall der Zona fasciculata vor, beim anderen (Fall 2) fand sich eine klinisch manifeste komplette Nebennierenrindeninsuffizienz. Beide Patienten haben einen beginnenden Hypogonadismus. Die neurologische Symptomatik ging im Fall 2 der Entwicklung der endokrinen Ausfälle voraus, im Fall 1 führten Familienanamnese und Nebennierenrindeninsuffizienz zur Diagnosestellung. Beim Nachweis einer peripheren Neuropathie bei männlichen Jugendlichen oder jüngeren Erwachsenen sollte auf die Zeichen einer beginnenden Nebennierenrindeninsuffizienz geachtet werden.
\end{abstract}

Adrenomyeloneuropathy, a rare cause of primary adrenal cortical insufficiency

Inherited via the $\mathrm{X}$ chromosome, adrenomyeloneuropathy is a rare cause of primary adrenocortical insufficiency. Neurological signs are of central and peripheral demyelinization, while endocrinologically it is characterized by Addison's disease and primary testicular insufficiency. In two patients with this condition the metabolic defect in the breakdown of longchain fatty acids was confirmed by an increased hexakosan (C 26) blood level. One patient had an isolated failure of the zona fasciculata; in the other there was clinically manifest complete adrenocortical insufficiency. Both patients had incipient hypogonadism. In the second case, neurological symptoms preceded the endocrinological ones, while in the first both the family history and the adrenocortical insufficiency led to the diagnosis. In peripheral neuropathy in a young male, attention should always be given to signs of incipient adrenocortical insufficiency.
Das Krankheitsbild der Adrenomyeloneuropathie $(\mathrm{AMN})$ ist eine im frühen Erwachsenenalter manifest

Dtsch. med. Wschr. 111 (1986), 1519-1522

(c) 1986 Georg Thieme Verlag Stuttgart - New York werdende Variante der Adrenoleukodystrophie (ALD), eines in der Pädiatrie bekannten Krankheitsbildes mit Ausfallserscheinungen des Nervensystems und der Nebennierenrinde (12). Die neurologischen Störungen in Form von Demyelinisierungszeichen betreffen bei der 
Tab. 1. Hypophysär-adrenale Hormone bei zwei Patienten mit Adrenomyeloneuropathie

\begin{tabular}{|c|c|c|c|c|c|}
\hline & $0 \mathrm{~min}$ & $\begin{array}{l}60 \min \\
\text { nach ACTH }\end{array}$ & $0 \mathrm{~min}$ & $\begin{array}{l}60 \text { min } \\
\text { nach } A C T H\end{array}$ & Normbereich \\
\hline Cortisol & 67 & 56 & 45 & 42 & $70-250 \mathrm{ng} / \mathrm{ml}$ \\
\hline DHEA-S & 75 & - & 57 & - & $280 \pm 104 \mu \mathrm{g} / \mathrm{dl}$ \\
\hline 17-OH-Pregnenolon & 177 & 236 & 30 & 64 & $30-350 \mathrm{ng} / \mathrm{dl}$ \\
\hline DHEA & 175 & 170 & 97 & 82 & $522 \pm 256 \mathrm{ng} / \mathrm{dl}$ \\
\hline 17-OH-Progesteron & 97 & 97 & 28 & 42 & $43-220 \mathrm{ng} / \mathrm{dl}$ \\
\hline 21-Desoxycortisol & 0,3 & 1,7 & 6,4 & 4,4 & $1,2-13 \mathrm{ng} / \mathrm{dl}$ \\
\hline 11-Desoxycortisol & 0,2 & 0,4 & 0,08 & 0,07 & $0,1-0,3 \mu \mathrm{g} / \mathrm{d} 1$ \\
\hline 11-Desoxycorticosteron & - & 15,4 & 8,7 & 9,2 & $2-15 \mathrm{ng} / \mathrm{dl}$ \\
\hline Aldosteron & 3,6 & 7,8 & 1,6 & 1,0 & $2-10 \mathrm{ng} / \mathrm{d}$ \\
\hline 18-OH-Corticosteron & 15,6 & - & 7,6 & 10,8 & $11,5-55 \mathrm{ng} / \mathrm{dl}$ \\
\hline Renin & 0,65 & - & 15,8 & - & $0,3-2,0 \mathrm{ng} / \mathrm{ml} \cdot \mathrm{h}$ \\
\hline ACTH & 829 & - & 1067 & - & $<25-160 \mathrm{pg} / \mathrm{ml}$ \\
\hline
\end{tabular}

AMN vorwiegend das periphere Nervensystem, die ALD ist durch Befall des Zentralnervensystems gekennzeichnet. Die AMN wird ebenso wie die ALD X-chromosomal gebunden vererbt (7). Das typische klinische Erscheinungsbild der AMN ist eine langsam progrediente distale Polyneuropathie und spastische Paraparese, eine progrediente Nebenniereninsuffizienz sowie ein langsam progredienter Hypogonadismus (2, 3, 11, 13). Die Ursache der Störung ist letztlich unbekannt; vermutet wird ein Enzymdefekt im Abbau extrem langkettiger Fettsäuren; sie sind im Plasma, in Fibroblasten, in der weißen Substanz des Gehirns und in den Nebennierenrindenzellen vermehrt nachweisbar $(5,6,8)$. Zur Sicherung der Diagnose und zum Nachweis von Hemizygoten dient die Bestimmung von Hexakosansäure (C 26) im Plasma; sie ist deutlich erhöht(6).

Im folgenden berichten wir über zwei Patienten mit Adrenomyeloneuropathie, die wir im letzten Jahr untersuchten.

\section{Kasuistik}

Fall 1: In der Familienanamnese des 32jährigen Patienten waren auffällig ein Großvater mütterlicherseits, der an einer Rückenmarksentzündung gestorben war, und ein Neffe, der mit 11 Jahren an einer schweren Form der Adrenoleukodystrophie erkrankte. Der Patient selbst klagte über ein seit 2 Jahren bestehendes, wechselnd starkes Bandagengefühl im Bereich der linken Wade. Seit einigen Monaten bestünden Miktionsstörungen in Form von imperativem Harndrang, wechselnd mit Harnverhalt und Inkontinenz. Seit etwa 10 Jahren bemerkte er einen langsamen Rückgang von Libido und Potenz, an der schon immer spärlichen Sekundärbehaarung seien keine Veränderungen aufgefallen. Zeitweilig bestünde eine deutliche Müdigkeit und Abgeschlagenheit.

Bei der klinischen Untersuchung fanden wir eine insgesamt deutliche Pigmentverstärkung des gesamten Integuments mit zusätzlicher fleckförmiger Pigmentierung im Bereich der Lippen und der Wangenschleimhaut. Die Sekundärbehaarung war spärlich ausgeprägt, zeigte jedoch das typisch männliche Verteilungsmuster. Die Testes waren mit $20 \mathrm{ml}$ beiderseits normal groß, hatten eine unauffällige Konsistenz und Oberfläche, waren jedoch beiderseits leicht druckschmerzhaft. Der Blutdruck lag mit $130 / 80 \mathrm{~mm} \mathrm{Hg}$ bei einer Pulsfrequenz von 68/min im Normbereich, aus der Vorgeschichte war keine Hypotoniesymptomatik und keine Hypotonie bekannt.

Die neurologische Untersuchung (4) ergab eine ganz diskrete Parese der Großzehenhebung links, die Bauchhautreflexe waren nicht auslösbar, der Quadricepsreflex eine Spur rechtsbetont. Das Babinskische Zeichen war beiderseits positiv. An den unteren Extremitäten bestand eine nach distal zunehmende strumpfförmige Sensibilitätsstörung für alle Qualitäten (ab Mitte Oberschenkel links und Mitte Unterschenkel rechts). Beim Rombergschen Versuch kam es zu ungerichtetem Schwanken bei Augenschluß. Die übrigen körperlichen Befunde der allgemein-internistischen sowie der neurologischen Untersuchung waren unauffällig. Motorische und sensible Nervenleitgeschwindigkeiten waren sowohl an den unteren als auch an den oberen Extremitäten deutlich verlangsamt. Die visuell evozierten Potentiale sowie die akustisch evozierten Stammpotentiale zeigten verlängerte Latenzen.

Laboratoriumsbefunde: Blutsenkungsreaktion, Blutbild, Differentialblutbild, Blutzucker, Leber- und Nierenwerte, Blutgasanalyse, Serumelektrolyte, insbesondere Natrium mit $142 \mathrm{mmol} / \mathrm{l}$ und Kalium mit 4,3 mmol/1, lagen im Normbereich.

Tab. 2. Hypophysär-testikuläre Hormone bei zwei Patienten mit Adrenomyeloneuropathie

\begin{tabular}{|l|c|c|c|}
\hline & Fall 1 & Fall 2 & Normbereich \\
\hline Testosteron & 199 & 179 & $200-1000 \mathrm{ng} / \mathrm{dl}$ \\
Dihydrotestosteron & 99 & 55 & $45 \pm 68 \mathrm{ng} / \mathrm{dl}$ \\
LH & 8,9 & 4,6 & $2-20 \mathrm{mU} / \mathrm{ml}$ \\
LH nach LHRH & 30 & 37,9 & - \\
FSH & 2,9 & 2,8 & $2-8 \mathrm{mU} / \mathrm{ml}$ \\
\hline
\end{tabular}

Glucocorticoide, Mineralocorticoide und deren Vorstufen wurden im Serum und Urin basal sowie nach ACTH-Injektion und Metopirontest bestimmt (Tabellen 1 und 2). Cortisol und Glucocorticoidvorstufen waren basal erniedrigt bzw. im unteren Normbereich und stiegen im ACTH-Kurztest nicht an. Die Ausscheidung des freien Cortisols war entsprechend vermindert. ACTH wurde 
Tab. 3. Meßwerte der langkettigen Fettsäuren C22-C26. (Die Messungen wurden im J. F. Kennedy Institute, Baltimore/Md., durchgeführt.)

\begin{tabular}{|l|c|c|c|c|c|}
\hline & Fall 1 & Fall 2 & Normalwerte & $\begin{array}{c}\text { Hemizygote } \\
\text { ALD/AMN }\end{array}$ & $\begin{array}{c}\text { Heterozygote } \\
\text { ALD/AMN }\end{array}$ \\
\hline C22 (\% Gesamtfettsäuren) & 0,665 & 0,671 & $1,18 \pm 0,61$ & $1,63 \pm 0,58$ & $1,33 \pm 0,54$ \\
C24 (\% Gesamtfettsäuren) & 0,987 & 1,030 & $0,78 \pm 0,32$ & $1,73 \pm 0,76$ & $1,90 \pm 0,80$ \\
C25 (\% Gesamtfettsäuren) & 0,044 & 0,049 & $0,03 \pm 0,03$ & $0,12 \pm 0,06$ & $0,20 \pm 0,34$ \\
C26 ( $\mathrm{g} / \mathrm{ml})$ & 1,429 & 1,514 & $0,33 \pm 0,18$ & $1,62 \pm 0,87$ & $0,82 \pm 0,32$ \\
C24 / C22 & 1,486 & 1,534 & $0,84 \pm 0,08$ & $1,63 \pm 0,20$ & $1,20 \pm 0,24$ \\
C26 / C22 & 0,055 & 0,061 & $0,01 \pm 0,01$ & $0,07 \pm 0,02$ & $0,04 \pm 0,02$ \\
\hline
\end{tabular}

deutlich erhöht gemessen. 18-Hydroxycorticosteron und Aldosteron zeigten Werte im unteren Normbereich, die Plasma-Reninaktivität lag im Normbereich. Im Metopirontest fand sich ein unzureichender Anstieg von 11-Desoxycortisol (Substanz S) nach Metopirongabe.

Der Serumtestosteronspiegel fand sich leicht vermindert, die basalen Gonadotropinspiegel und die stimulierten Werte lagen im Normbereich.

Die Analyse der langkettigen Fettsäuren C22 bis C 26 zeigten den typischen Befund für die ALD/AMN mit deutlicher Erhöhung der Hexakosansäure (Tabelle 3).

Fall 2: Dieser Patient hatte bereits seit dem Kindesalter eine braune Hautfarbe. Seine körperliche Leistungsfähigkeit, zum Beispiel beim Schulsport, war schon immer leicht eingeschränkt. Im April 1980 entwickelte sich nach einem Zeckenbiß eine Kopfschwartenphlegmone, die chirurgisch und antibiotisch behandelt wurde. Gegen Ende des Klinikaufenthaltes bemerkte der Patient eine Gehstörung; unter der Verdachtsdiagnose Enzephalomyelitis disseminata wurde eine Behandlung mit ACTH-Infusionen durchgeführt. Im weiteren Verlauf entwickelten sich eine zunehmende körperliche Schwäche und Hypotonie. Im November 1984 stellte sich der Patient dann erstmals in unserer Abteilung vor.

Bei der klinischen Untersuchung fand sich eine deutliche Pigmentverstärkung, vor allem an den sonnenexponierten Hautstellen. Der Bartwuchs war spärlich, die Axillarbehaarung fehlte, die Pubesbehaarung war horizontal begrenzt. Lungen und Herz waren auskultatorisch unauffällig. Die Herzaktion war regelmäßig mit einem Puls von $80 / \mathrm{min}$. Der Blutdruck betrug im Liegen 100/70 $\mathrm{mm} \mathrm{Hg}$, im Sitzen 130/80 mm Hg. Das Abdomen war unauffällig, Leber und Milz waren nicht vergrößert tastbar. Das äußere Genitale war unauffällig mit einem Hodenvolumen von beiderseits $20 \mathrm{ml}$. Es lag eine deutliche Atrophie der Oberschenkelmuskulatur beiderseits und ein Spitzfuß beiderseits vor. Bei der neurologischen Untersuchung fand sich eine hochgradige spastische Tonussteigerung mit mäßiger, distal betonter Parese der Beine. Sämtliche Muskeleigenreflexe waren seitengleich und bis auf die lediglich schwach auslösbaren Achillessehnenreflexe lebhaft. Das Babinskische Zeichen war beiderseits positiv. Weiter bestanden eine Hypalgesie, pathologische Zahlendiskrimination und ein abgeschwächtes Vibrationsempfinden an beiden Füßen. Die Lageempfindlichkeit bei geführten Großzehenbewegungen war pathologisch.

Elektrophysiologisch fand sich an den unteren Extremitäten eine hochgradige Verzögerung der Nervenleitgeschwindigkeit. Im Elektromyogramm waren Zeichen eines neurogenen Umbaus als Ausdruck einer chronisch-neurogenen Schädigung im Musculus tibialis anterior links nachweisbar. Die visuellen und akustisch evozierten Potentiale sowie die zentrale Überleitungszeit der sensibel evozierten Potentiale waren regelrecht.

Laboratoriumsbefunde: Die Routine-Laboratoriumsparameter waren bis auf eine leichte Erhöhung der $\gamma$-GT unauffällig. Die hypophysär-adrenalen sowie die hypophysär-testikulären Hormone sind in den Tabellen 1 und 2 dargestellt. Gluco- und Mineralocorticoide sowie deren Vorstufen waren basal erniedrigt und zeigten keine Stimulierbarkeit nach ACTH. Der ACTH- und Reninspiegel wurde stark erhöht gemessen. Die basalen und stimulierten Gonadotropinspiegel lagen im Normbereich, Testosteron fand sich leicht vermindert. Der Hexakosansäure-Spiegel (C 26) lag für eine Adrenomyeloneuropathie im typischen Bereich (Tabelle 3).

\section{Diskussion}

Bei beiden Patienten belegen die Ergebnisse der Fettsäurenanalysen, die neurologischen sowie die endokrinologischen Befunde die Diagnose einer Adrenomyeloneuropathie. In beiden Fällen wies jedoch der Ausprägungsgrad sowohl der endokrinen als auch der neurologischen Symptomatik deutliche Unterschiede auf.

Im Fall 1 liegt eine beginnende Nebennierenrindeninsuffizienz vor. Hierfür sprechen der mangelnde Anstieg des Cortisols, der deutlich erniedrigte Dehydroepiandrosteronsulfat-Spiegel, der deutlich erhöhte ACTH-Spiegel und die verminderte Ausscheidung von freiem Cortisol. Die im unteren Normbereich liegenden 18-Hydroxycorticosteron- und Aldosteron-Spiegel belegen, daß noch keine manifeste Störung der Mineralocorticoidsynthese vorliegt. Diese Befunde werden durch die unauffälligen Elektrolytwerte, die normale Plasma-Reninaktivität und den unauffälligen Blutdruck ergänzt. Neben der Glucocorticoid-Substitutionstherapie ist somit zum jetzigen Zeitpunkt keine Mineralocorticoidtherapie nötig.

Im Fall 2 besteht eine komplette manifeste Nebennierenrindeninsuffizienz: Gluco- und Mineralocorticoide sind basal erniedrigt, und die Steroidmetabolite im Urin sind vermindert, die Plasma-Reninaktivität ist erhöht, die adrenale Reserve ist deutlich eingeschränkt.

Aus den Basal- und Stimulations-Werten der Steroidvorstufen im ACTH-Kurztest ergibt sich bei beiden Patienten kein Anhalt für einen Enzymdefekt, wie ihn Allen und Mitarbeiter (1) als Ursache des bei einem ihrer Patienten deutlich erhöhten 11-Desoxycortisolspiegels (11-Hydroxylasemangel) annehmen. Bei unseren beiden Patienten lagen die 11-Desoxycortisol- und 11-Desoxycorticosteron-Spiegel basal im Normbereich und ließen keine überschießende Stimulierbarkeit nach ACTH-Gabe erkennen.

Weiterhin fanden wir weder einen Hinweis auf einen $3 \beta$-Hydroxysteroiddehydrogenase-Mangel (normale basale und stimulierte Pregnenolon- und Dehydroepiandrosteron-Spiegel) (1) noch auf einen 21-Hydroxylase- 
Mangel (normale basale und stimulierte 17-Hydroxyprogesteron- und 21-Desoxycortisol-Spiegel).

Ursache dieser Störungen ist möglicherweise eine Ablagerung der in höheren Konzentrationen zytotoxisch wirkenden Fettsäuren in den Nebennierenrindenzellen (13). Ein primär partieller Befall von Zona fasciculata und Zona reticularis mit Aussparung der Zona glomerulosa wird beschrieben, aber auch der typische Morbus Addison mit Ausfall aller drei Zonen der Nebennierenrinde ist bekannt (3).

Die Ablagerungen der langkettigen Fettsäuren sind nicht nur in Nebennierenrindenzellen nachweisbar, sondern auch in den Testes (9). Der bei beiden Patienten anamnestisch angegebene Libido- und Potenz-Verlust zusammen mit den erniedrigten Testosteronspiegeln deutet auf einen Hodenschaden hin. Auffällig und uns bisher nicht erklärbar ist bei beiden Patienten eine Normalisierung des Testosteron-Spiegels unter Cortisonsubstitutionstherapie; eine Besserung von Libido und Potenz trat jedoch nicht ein.

Die Manifestation der Nebennierenrindeninsuffizienz kann dem neurologischen Krankheitsbild vorausgehen oder nachfolgen. Im Fall 1 wurde bei bekannter Erkrankung von Familienmitgliedern gleichzeitig mit dem Morbus Addison die diskrete neurologische Symptomatik entdeckt. Im Fall 2 hatte sich über Jahre eine spastische Paraparese entwickelt, der Morbus Addison wurde 4 Jahre nach dem neurologischen Krankheitsbild diagnostiziert.

\section{Folgerungen}

Beim Nachweis einer peripheren Neuropathie bei Jugendlichen oder jüngeren männlichen Erwachsenen sollte auf die zum Teil milden Zeichen einer Nebennierenrindeninsuffizienz geachtet werden. Eine Einschränkung der adrenalen Reserve läßt sich im Frühstadium häufig nur über eine ACTH-Belastung diagnostizieren. Bei Patienten mit Morbus Addison und zusätzlichen neurologischen Symptomen ist an die Möglichkeit des geschilderten Krankheitskomplexes zu denken. Die Untersuchung der Blutsverwandten von Erkrankten eröffnet Wege zur Frühdiagnose und genetischen Bera- tung. Die Sicherung der Diagnose Adrenomyeloneuropathie ist durch eine Analyse der langkettigen Fettsäuren möglich. Das Phänomen des Testosteronanstiegs nach Einsetzen der Glucocorticoidsubstitution verdient weitere Aufmerksamkeit.

\section{Literatur}

(1) Allen, J. P., T. Kepic, D. Garwacki, M. Yunus: Adrenal defect in adrenomyelodystrophy. Sth. med, J. (Bgham, Ala.) 75 (1982), 877.

(2) Davis, L. E., R. D. Snyder, D. N

Orth, W. E. Nicholson, M. Kornfeld, D.

F. Seelinger: Adrenoleukodystrophy and adrenomyeloneuropathy associated with partial adrenal insufficiency in three generations of a kindred. Amer. J. Med. 66 (1979), 342 .

(3) Griffin, J. W., E. Goren, H. Schaum. berg, W. K. Engel, L. Loriaux: Adrenomyeloneuropathy: a probable variant of adrenoleukodystrophy. Neurology (Minneap.) 27 (1977), 1107

(4) Krause, K. H., K. Brosi, M. Rittmann, K. Schleglmann, K. Frank, R. Ziegler: EMG und evozierte Potentiale bei der Adrenomyeloneuropathie. EEG - EMG 16 (1985), 101.

(5) Moser, H. W., A. B. Moser, N. Kawamura, J. Murphy, K. Suzuki, H. Schaumberg, Y. Kishimoto: Adrenoleukodystrophy. Elevated C 26 fatty acid in cultured skin fibroblasts. Ann. Neurol. 7 (1980), 542 .

(6) Moser, H. W., A. B. Moser, K. K. Frayer, W. Chen, J. D. Schulman, B. P. O'Neill, Y. Kishimoto: Adrenoleukodystrophy. Increased plasma content of saturated very long chain fatty acids. Neurology (Minneap.) 31 (1981), 1241.

(7) O'Neill, B. P., L. C. Marmion, E. R.

Feringa: The adrenoleukomyeloneuropathy complex. Expression in four generations. Neurology (Minneap.) 31 (1981), 151.

(8) O'Neill, B. P., H. W. Moser, L. C. Marmion: Adrenoleukodystrophy. Elevated C 26 fatty acid in cultured skin fibroblasts and correlation with disease expression in three generations of a kindred. Neurology (Minneap.) 32 (1982), 540.

(9) Powers, J. M., H. H. Schaumberg: The testis in adreno-leukodystrophy. Amer. J. Path. 102 (1981), 90.

(10) Rosenfield, R. L., B. H. Rich, J. I. Wolfsdorf, F. Cassorla, J. S. Parks, A. M.

Bongiovanni, C. H. L. Shackleton: Pubertal presentation of congenital $\Delta 5-3 \beta$. hydroxysteroid dehydrogenase deficiency. J. clin. Endocr. 51 (1980), 345.

(11) Scully, R. E., E. J. Mark, B. U.

McNeely: Case records of the Massachusetts General Hospital. New Engl. J. Med. 306 (1982), 286.

(12) Schaumberg, H. H., J. M. Powers, C. S. Raine, K. Suzuki, E. P. Richardson Adrenoleukodystrophy. Arch. Neurol. (Chic.) 32 (1975), 577.

(13) Schaumberg, H. H., J. M. Powers, C. S. Raine, P. S. Spencer, J. W. Griffin, J. W. Prineas, D. M. Boehme: Adrenomyeloneuropathy: a probable variant of adrenoleukodystrophy. Neurology (Minneap.) 27 (1977) 1114 .

Dr. K. Frank, Dr. O. Schrecker, Prof. Dr. R. Ziegler

Abteilung für Endokrinologie

Medizinische Universitätsklinik

6900 Heidelberg 1, Luisenstr. 5

Dr. K. Brosi, Privatdozent Dr. K. H. Krause

Neurologische Universitätsklinik

6900 Heidelberg 1, Voßstr. 2

Prof. Dr. P. Vescei

Pharmakologisches Institut der Universität 6900 Heidelberg 1, Im Neuenheimer Feld 366

Prof. H. W. Moser, M. D.

John F. Kennedy Institute für Handicapped Children Baltimore, MD, U. S. A. 